\title{
Treatment dilemma of a depressed patient with metabolic syndrome
}

\begin{abstract}
Background: Depression affects around 121 million people world wide according to the world health organization (WHO) and is the second leading cause of disability globally. Metabolic syndrome is defined as a combination of conditions that predispose individuals to several diseases, mainly cardiovascular diseases. The association between metabolic syndrome and depression represents a major social issue for its increasing prevalence. However, proper treatment strategies for patients who have the two overlapping conditions are not yet established.
\end{abstract}

Case report: We report a challenging but successful treatment strategy of a 60 year old male, with metabolic syndrome, diagnosed with depression.

Aim: The aim of the report is to highlight the need of developing evidence based treatment strategies for patients with depression overlapping with metabolic syndrome.

Conclusion: Treating patients with depression and metabolic syndrome requires a multidisciplinary team, and cautious decisions as to which antidepressant to start in order to avoid deterioration in their comorbidities. More research is needed in this field as both metabolic syndrome and depression are increasing in prevalence and are among the leading causes of burden to the healthcare system.

Keywords: depression, metabolic syndrome, antidepressant, psychopharmacology, anxiety

\section{Introduction}

Depression is a disorder characterized by emotional, cognitive and somatic symptoms that affect physical health hence increasing morbidity and mortality. The disorder affects around 121 million people worldwide (WHO) and is the second leading cause of disability globally. Depressed patients have a 4-fold increase in risk of early death in comparison to their peers without depression. Older adults with depressive symptoms have poorer functioning compared to those with chronic medical conditions such as lung disease, hypertension or diabetes (WHO, 2016) ${ }^{1,2}$ In particular, mortality studies indicate that cardiovascular diseases (CVDs) are the most common causes of premature death in depressed patients. ${ }^{3,4}$ The association between depression and CVD is thought to be mediated by the so-called metabolic syndrome (MeS).

Metabolic syndrome is defined as a combination of conditions that predispose individuals to several diseases, mainly cardiovascular diseases. It is characterized by increased abdominal obesity, increased triglycerides, decreased high-density lipoprotein cholesterol (HDL-C), high blood pressure (BP) and high fasting plasma glucose (FPG) or diabetes. The prevalence of the condition ranges between $11 \%$ to $43 \%$ (WHO) and peaks around 60-75 years.

Epidemiological data and meta-analyses of cross-sectional and cohort studies published up to January 2012 indicate a bidirectional association between depression and MeS. The association between metabolic syndrome and depression represents a major social issue for its increasing prevalence. However, proper treatment strategies for patients who have the two overlapping conditions are not yet established, and managing a patient with depression and metabolic syndrome presents a clinical dilemma; mainly because severe psychotropic drugs are actually associated with adverse metabolic effects.
Volume 9 Issue 3 - 202 I

\author{
Khalid Al Jalahma,' Najib AI Qsous² \\ 'Psychiatric Resident, Bahrain \\ ${ }^{2}$ Psychiatry Specialist, Jordan
}

Correspondence: Dr. Khalid Al Jalahma, MBChB, Psychiatric Resident, Bahrain, Email dr_khalid89@hotmail.com

Received: June 30, 2021 | Published: July 28, 2021

\section{Case report}

We report a 60 year old Jordanian Male patient, known case of diabetes mellitus (15years), hypertension (10years) and dyslipidemia. The patient presented to the psychiatric clinic of Bahrain Defence force Hospital on Tuesday 17/05/2019 with a 2 month history of low mood associated with fatigue, insomnia, anhedonia, poor concentration, decreased libido, anxiety and loss of appetite along with social isolation and death wishes, but no active suicidal thoughts or ideations. He also denied any previous history of suicidal attempts or self harm. History was negative for hallucinations, and manic symptoms were also ruled out.

The patient also reported history of losing $10 \mathrm{kgs}$ since the onset of his symptoms and he linked the onset of his symptoms to his retirement, as he was asymptomatic prior to it.

Medication history: metformin and Gliclazide. atenolol, furosemide, simvastatin Social history: Patient does not smoke, does not consume alcohol or use any recreational drugs. Married and a father to 5 children, previously working as an engineer and retired for 3years. General physical and systemic physical examination was unremarkable.

Mental state examination: a middle aged man, average built, appropriately dressed for age, gender and weather. Patient's eye to eye contact was downcasting with limited spontaneous body movement. He had restricted facial expression with an unshaved untidy beard and hair. No abnormal behavior or posture was noted. His speech was logical with normal rate, rhythm and volume. His mood was low with restricted mood congruent affect. The thought content of the patient was depressive in nature, and included a sense of hopelessness, worthlessness and helplessness. There were no suicidal thoughts, delusions or obsessions. There were no abnormal perceptual experiences. His cognitive function was intact during examination. 
Investigations: baseline investigations were done including complete blood count (CBC), Urea and electrolytes (U\&E), Liver function test (LFT), Renal function test (RFT) and Electrocardiogram (ECG) which were all normal. His blood sugar level was controlled with a fasting blood sugar reading of $6.7 \mathrm{mmol} / \mathrm{L}$.

Beck's depression inventory was used and the patient scored 24 which is considered moderate depression. Hamilton Anxiety rating scale was also used and the patient scored 22 which is considered anxiety of mild to moderate severity.

Management: Patient was first started on paroxetine, which is a Selective Serotonin Reuptake inhibitor (SSRI). This class is recommended as a first line treatment for depression. However, the patient could not tolerate it and developed severe initial anxiety including insomnia and loss of appetite. Hence, he was not compliant and stopped it after 2 weeks.

Another drug was then started; fluoxetine which is the least likely SSRI to cause initial anxiety. Patient's mood improved with fluoxetine for 2 months after which he developed severe hyponatremia and required hospitalization. Hence the drug was stopped.

The patient was then started on venlafaxine, which is a Selective Norepinephrine Reuptake inhibitor (SNRI) for 2 months until he developed severe uncontrolled hypertension and venlafaxine was stopped.

Patient was finally started on mirtazapine which is a Norepinephrine and Serotonin Specific Antagonist (NaSSA). The patient's condition stabilized on mirtazapine; his depressive symptoms subsided and his metabolic status was also stable. The patient was on medication for a total of 9 months and was symptom free at 3 months.

\section{Discussion}

The aim of this case report is to highlight the challenges associated with treating depressive patients with associated metabolic syndrome; keeping in mind the considerable metabolic effects antidepressant medications have. The aim is to also suggest that although SSRIs are considered first line in the treatment of depression. Factors like associated metabolic syndrome should be considered.

A cross sectional study comparing Paroxetine, sertraline, fluoxetine, fluvoxamine and citalopram concluded that SSRI's as a group were significantly associated with Type 2 diabetes mellitus, hypercholesterolemia and abdominal obesity. Paroxetine showed the strongest association with obesity and Diabetes Mellitus Type 2.

Another Double-blind, randomized trial assessed body weight changes during treatment with SSRIs over 26-32 weeks. It concluded that patients treated with paroxetine had a significant weight change while those treated with sertraline had a non-significant weight gain and patients treated with fluoxetine had weight loss.

SSRI's are also frequently linked to Dyslipidemia. According to a 16-week follow-up, Beyazyuz et al study comparing SSRIs including fluoxetine, paroxetine, sertraline, and citalopram in how they affect the lipid profile. It concluded that all of them raise Triglycerides and Low Density Lipoprotein (LDL) levels.

On the other hand, a prospective, controlled study design aimed to investigate the influence of mirtazapine treatment on body weight, body fat mass and glucose metabolism. The study concluded that Mirtazapine has a significant increase in body weight but it did not influence the glucose homeostasis. ${ }^{5-18}$

\section{Conclusion}

A growing body of evidence shows that depression is related to an increased risk of diabetes and cardiovascular disease and vice versa. Hence early detection and treatment of metabolic syndrome is a key element of avoiding secondary depression.

Treating patients with depression and metabolic syndrome requires a multidisciplinary team, and cautious decisions as to which antidepressant to start in order to avoid deterioration in their comorbidities. More research is needed in this field as both metabolic syndrome and depression are increasing in prevalence and are among the leading causes of burden to the healthcare system.

\section{Acknowledgments}

None.

\section{Funding}

None.

\section{Conflicts of interest}

The authors declare no conflict of interest.

\section{References}

1. Sadock BJ, Sadock VA, Ruiz P. Kaplan \& sadock's synopsis of psychiatry behavioral sciences/clinical psychiatry. Wolters Kluwer, New York, Eleventh edition; 2015.

2. Ghari Pour M, Baghei A, Boshtam M, et al. Prevalence of metabolic syndrome among the adults of central of areas of Iran (as part of" Isfahan Healthy Heart Study"). J Birjand Univ Med Sci. 2006;13(3):9-15.

3. Osby U, Brandt L, Correia N, et al. Excess mortality in bipolar and unipolar disorder in Sweden. Arch Gen Psychiatry. 2001;58(9):844-850.

4. Kinder LS, Carnethon MR, Palaniappan LP, et al. Depression and the metabolic syndrome in young adults: findings from the Third National Health and Nutrition Examination Survey. Psychosom Med. 2004;66:316322.

5. Noorbala AA, Alipour A, Shaghaghi F, et al. The effect of emotional disclosure by writing on depression severity and defense mechanisms among depressed patients. Tehran Med Sci Univ. 2011;18(93):1-10.

6. Malhotra N, Kulhara P, Chakrabarti S, et al. A prospective, longitudinal study of metabolic syndrome in patients with bipolar disorder and schizophrenia. J Affect Disord. 2013;150(2):653-658.

7. Chokka P, Tancer M, Yeragani VK. Metabolic syndrome: relevance to antidepressant treatment. J Psychiatry Neurosci. 2006;31:414.

8. Simon GE, Ludman EJ, Linde JA, et al. Association between obesity and depression in middle- aged women. Gen Hosp Psychiatry. 2008;30:3239.

9. Goldbacher EM, Bromberger J, Matthews KA. Lifetime history of major depression predicts the development of the metabolic syndrome in middle-aged women. Psychosom Med. 2009;71(3):266-272.

10. Miettola J, Niskanen LK, Viinamaki H, et al. Metabolic syndrome is associated with self-perceived depression. Scand J Prim Health Care. 2008;26(4):203-210.

11. McCaffery JM, Niaura R, Todaro JF, et al. Depressive symptoms and metabolic risk in adult male twins enrolled in the National Heart, Lung, and Blood Institute twin study. Psychosom Med. 2003;65(3):490-497.

12. Bonnet, F, Irving, K, Terra, JL, et al. Anxiety and depression are associated with unhealthy lifestyle in patients at risk of cardiovascular disease. Atherosclerosis. 2005;178(2):339-344. 
13. Herva A, Räsänen P, Miettunen J, et al. Co-occurrence of metabolic syndrome with depression and anxiety in young adults: the Northern Finland 1966 Birth Cohort Study. Psychosom Med. 2006;68(2):213-216.

14. Repousi N, Masana MF, Sanchez-Niubo A, et al. Depression and metabolic syndrome in the older population: A review of evidence. Journal of Affective Disorders. 2018; 237:56-64.

15. Marazziti D, Rutigliano G, Baroni S, et al. Metabolic syndrome and major depression. CNS Spectrums. 2013;19(4):293-304.
16. Depression and other common mental disorders: Global Health Estimates. Geneva: World Health Organization; 2017.

17. Olfson M, Marcus SC, Pincus HA, et al. Antidepressant prescribing practices of outpatient psychiatrists. Arch Gen Psychiatry. 1998;55:310 316.

18. Beyazyüz M, Albayrak Y, Eğilmez OB, et al. Relationship between SSRIs and metabolic syndrome abnormalities in patients with generalized anxiety disorder: a prospective study. Psychiatry investigation. 2013. 